\title{
Um estudo analítico da dinâmica da decolagem e do pouso de aeronaves com forças dependentes da velocidade
}

(An analytical study of the dynamics of takeoff and landing in airplanes with velocity dependent forces)

\author{
C.C. Pellegrini ${ }^{\boxplus}$, M.S. Rodrigues \\ Departamento de Ciência Térmicas e dos Fluidos, Universidade Federal de São João del Rei, São João del Rei, MG, Brasil \\ Recebido em 5/1/2015; Aceito em 9/3/2015; Publicado em 30/6/2015
}

\begin{abstract}
Neste trabalho a decolagem e pouso de uma aeronave são analisados através da da Segunda Lei de Newton. Ambos os processos são sucintamente revisados. Como resultado, são obtidas expressões analíticas para as distâncias percorridas em pista e suas dependências com o peso da aeronave, sua curva de potência, a configuração aerodinâmica, o atrito de rolamento e as condicões atmosféricas. Características matemáticas das soluções são estudadas, para efeitos didáticos. Os resultados obtidos pela análise são comparados com dados de decolagem obtidos da literatura para uma aeronave comercial e com dados obtidos em testes realizados com um veículo aéreo não tripulado de pequeno porte, construído nos laboratórios da universidade. Em ambos os casos, as soluções analíticas obtidas mostram-se bastante precisas.
\end{abstract}

Palavras-chave: corrida de decolagem e pouso, engenharia aeronáuitca.

In this work, the takeoff and landing of an airplane are studied through Newton's second law. Both processes are briefly revised. As a result, analytical expressions are obtained for the distances travelled in the airstrip and its dependences with the airplane weight, its power curve, the aerodynamic configuration, the rolling resistance and the atmospheric conditions. Mathematical features of the solutions are studied for didactical purposes. The results yielded by the analysis are compared with takeoff data obtained in the literature, for a commercial airplane, and with experimental data, obtained in the university laboratory for a small size unmanned aerial vehicle. In both cases, the analytical solutions obtained are considerably accurate.

Keywords: takeoff and landing, aeronautical engineering.

\section{Introdução}

Qual o comprimento de pista necessário para se decolar e pousar uma aeronave, conhecidos seu peso, curva de potência, configuração aerodinâmica, atrito de rolamento e condições atmosféricas? Essa é uma pegunta essencial tanto no projeto quanto na operação da aeronave. Todos os textos clássicos em engenharia aeronáutica oferecem alguma resposta a essa pergunta, mas o problema ainda guarda aspectos interessantes do ponto de vista didático.

Para os autores, a pergunta - e sua resposta tornaram-se importantes quando eles se envolveram com o projeto de um VANT cargueiro (ver Apêndice A para uma descrição sucinta dos termos aeronáuticos), de pequeno porte, destinado a participar da competição SAE Aerodesign [प]. Os estudantes inscritos formam equipes que representam a Instituição de Ensino Superior à qual estão ligados. As equipes são desafiadas anualmente com novos regulamentos baseados em desafios enfrentados pela indústria aeronáutica. Para que o evento não se transforme em uma competição entre marcas comerciais de aeromodelos, severas limitações são impostas à potência do motor e à geometria da aeronave. Quase sempre, entretanto, necessita-se saber se o comprimento de pista disponível é suficiente para a carga a ser transportada.

Este é, portanto, o objetivo do presente estudo: desenvolver expressões analíticas para as distâncias de decolagem e pouso de uma aeronave, seja ela um modelo comercial existente ou um VANT de pequeno porte. Até o limite do nosso conhecimento, tal solução é inédita na literatura sob forma analítica em um caso

\footnotetext{
${ }^{1}$ E-mail: pelle@ufsj.edu.br.

${ }^{2}$ A competição SAE Aerodesign "é um desafio lançado aos estudantes de Engenharia que tem como principal objetivo propiciar a difusão e o intercâmbio de técnicas e conhecimentos de Engenharia Aeronáutica entre estudantes e futuros profissionais da engenharia da mobilidade, através de aplicações práticas e da competição entre equipes".

Copyright by the Sociedade Brasileira de Física. Printed in Brazil.
} 
tão geral quanto o aqui analisado. No desenvolvimento, especial atenção é dedicada aos limites de aplicabilidade dos modelos matemáticos utilizados e, portanto, da própria solução obtida.

Os resultados obtidos são comparados com medidas preliminares realizadas com um dos protótipos de VANT construídos pela equipe e com dados obtidos da literatura para uma aeronave comercial, o Cessna 172 Skyhawk.

\section{Análise da decolagem}

Nesta seção, primeiramente o processo de decolagem é descrito. Em seguida, os resultados disponíveis na literatura são brevemente explicados e a equação do movimento é resolvida e analisada matematicamente.

\subsection{O processo de decolagem}

As forças atuantes em uma aeronave durante o processo de decolagem são ilustradas na Fig. 凹. Nesta, $T$ é a tração da hélice, $F_{A}$ é a força de arrasto (Apêndice A), $F_{S}$ a força de sustentação (Apêndice A), $W$ é o peso da aeronave e $R$ é a força de atrito de rolamento entre os trens de pouso e o pavimento. Todas, exceto o peso, dependem da velocidade da aeronave.

Decolar uma aeronave é essencialmente acelerá-la do repouso até uma velocidade $V_{d}$ em que $F_{S} \geq W$. Possuindo um grupo moto-propulsor (GMP - Apêndice A) capaz de proporcionar uma tração $T>F_{A}+R$ no intervalo $0 \leq V \leq V_{d}$, teoricamente trata-se apenas de dispor do comprimento de pista necessário ${ }^{\mathbf{B}}$ para atingir $V_{d}$.

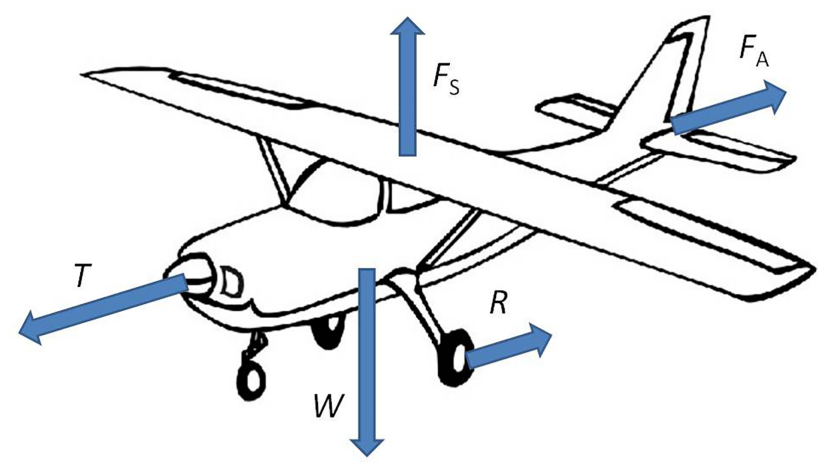

Figura 1 - Forças atuantes durante a decolagem.

Na prática, a rotina é ligeiramente mais complicada. A força de sustentação gerada pelas asas depende do ângulo de ataque (Apêndice A). A Fig. 叉, reproduzida da Ref. [0], mostra esta dependência através da varição do coeficiente de sustentação (Apêndice A), $C_{S}$, com o ângulo de ataque, $\alpha$. Fica claro que $C_{S}$ aumenta com $\alpha$ até um valor máximo e depois cai bruscamente. Este ponto é conhecido como ponto de estol (Apêndice A) e sinaliza a rápida perda de sustentação da aeronave, que geralmente entra em situação de risco.

No projeto padrão das aeronaves, as asas são montadas na fuselagem alinhadas ou praticamente alinhadas em relação à sua linha de centro. Com isso, a aeronave correrá na pista com a mínima força de arrasto, que ocorre quando $\alpha \approx 0$, como mostra a Fig. $\square$, que também ilustra o comportamento desta força através do coeficiente de arrasto, $C_{A}$. Para decolar, acelera-se com $\alpha \approx 0$ até uma velocidade em que a força de sustentação máxima, obtida com $\alpha_{\max }$ e $C_{S \max }$, se iguale ao peso e, um pouco depois deste momento (por razões de segurança), levanta-se o nariz da aeronave, em um movimento conhecido como arfagem (Apêndice A), momento em que ela decola.

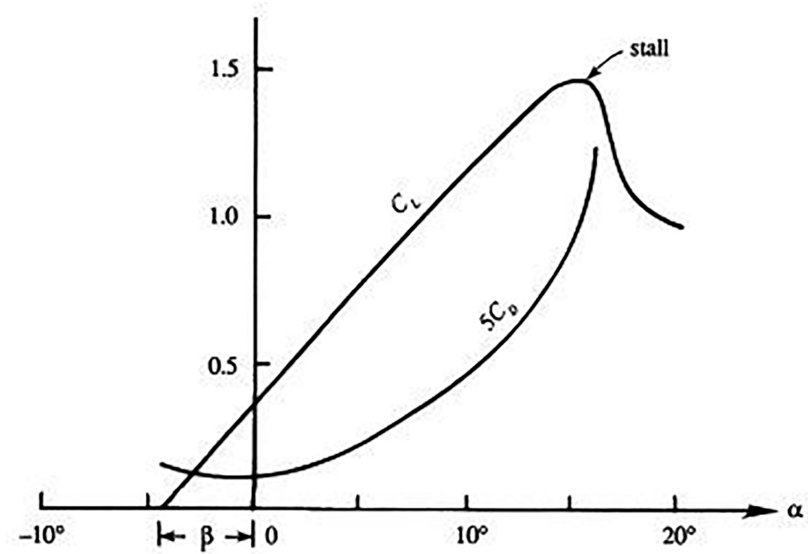

Figura 2 - Coeficientes genéricos de sustentação e arrasto em função do ângulo de ataque. Reproduzido da Ref. [च]].

Este procedimento é, sempre que possível, realizado contra o vento, de modo que a velocidade relativa entre o ar a aeronave aumente, aumentando a força de sustentação para uma dada velocidade em relação ao solo. Isso também aumenta a força de arrasto, o que não representa problema desde que se disponha de potência para garantir a condição $T>F_{A}+R$. Em geral, nossa análise indica e a prática aeronáutica confirma que a distância de decolagem efetivamente diminui com o aumento da velocidade do vento incidente.

\subsection{Revisão de literatura}

A análise clássica da corrida de decolagem parte do somatório de forças horizontais mostrada na Fig. 四 e obtém o valor da distância necessária como resultado de uma integral envolvendo estas forças. Estas, entretanto, dependem da velocidade em uma forma que, conforme a modelagem adotada, podem tornar a integral mais ou menos complicada.

A literatura aeronáutica tradicional [3], []], simplifica a integração utilizando valores médios para as forças, calculados a $70 \%$ da velocidade de decolagem. A sim-

\footnotetext{
${ }^{3}$ Como $F_{A}$ e $R$ são funções crescentes da velocidade enquanto $T$ é decrescente, nem sempre a condição é satisfeita e nem todo GMP é capaz de acelerar sua aeronave até a decolagem.
} 
plificação apresenta bons resultados práticos, mas obscurece o processo e apresenta diversas limitações conhecidas [0]. A mais importante do ponto de vista físico é o fato da integração aproximada não fornecer uma função $S=S(V)$.

$\mathrm{Na}$ Ref. [G] é apresentado um método estatístico de análise de corrida e decolagem. Tal método foi construído correlacionando estatisticamente dados experimentais do comprimento de pista necessário para se decolar diferentes modelos de aeronaves comerciais com seus peso, área alar (Apêndice A), potência do motor e coeficiente de sustentação. Mais uma vez, a dinâmica da decolagem permanece escondida.

Alguns textos [甘, [6] recomendam a resolução da integral por métodos numéricos, entendendo que a solução do problema per si é o bastante para as finalidades pretendidas na prática.

Apenas um dos autores pesquisados [0] apresenta solução analítica para a integral, mas sob uma forma particular da função $T=T(V)$ que não representa a realidade em muitos casos de interesse prático, conforme será visto adiante.

\subsection{A Equação do movimento}

Para uma aeronave em procedimento de decolagem, conforme ilustra a Fig. 四, a segunda lei de Newton escreve-se

$$
T-F_{A}-R=m \frac{d V}{d t}
$$

no intervalo $0 \leq V \leq V_{d}$, em que $V$ é a velocidade da aeronave relativa ao solo e $t$ o tempo.

Pela regra da cadeia, pode-se escrever $d V / d t=$ $(d V / d S)(d S / d t)=V d V / d S$, em que $S$ é o deslocamento. Com isto, pode-se integrar a Eq. (四) entre o início do movimento e o momento da decolagem e obter a forma integral da equação do movimento

$$
S_{d}=\frac{W}{g} \int_{0}^{V_{d}} \frac{V d V}{T-F_{A}-R},
$$

em que $S_{d}$ é a distância percorrida até a decolagem, $W$ o peso total da aeronave, $V_{d}$ é a velocidade de decolagem e todas as forças envolvidas dependem de $V$. O peso total foi considerado constante, pois o único elemento cujo peso poderia variar durante a decolagem é o combustível, cujo nível não se reduz significativamente.

Na presente análise, a tração da hélice será modelada de acordo com a quadrática

$$
T=a\left(V+U_{0}\right)^{2}+b\left(V+U_{0}\right)+T_{0},
$$

em que $a, b$ e $T_{0}$ são constantes, $U_{0}$ é a velocidade do vento frontal e $V+U_{0}$ é a velocidade da aeronave relativa ao vento. Matematicamente a equação anterior, e as posteriores que dependem de $V+U_{0}$, podem ser utilizadas para um vento de cauda tomando $U_{0}<0$, mas a prática não recomenda a decolagem em tais circunstâncias.

A constante $T_{0}$ é conhecida como a tração estática do GMP, pois é o valor observado com o GPM em repouso e sem vento $\left(V=U_{0}=0\right)$. O mesmo valor seria obtido com $V=-U_{0}$ mas em geral os testes não são realizados nessa circunstância.

O modelo adotado para a tração em GPMs a hélice é sugerido pela Ref. [U] $\operatorname{com} b=0$. O modelo aqui posposto é uma generalização necessária desta forma, pois a literatura aeronáutica indica [ $[$ ] e experimentos realizados em uma bancada desenvolvida pela própria equipe para motores elétricos confirmam que $b \neq 0$. Os experimentos mostraram também que a parábola apresenta concavidade para baixo, com $a<0$, um resultado que parece sempre válido para motores elétricos. Para motores a combustão interna a parábola geralmente apresenta concavidade para cima, com $a>0$, como foi comprovado ajustando uma quadrática aos dados obtidos na Ref. [8]. Em ambos os casos, é evidente que a tração deve diminuir monotonicamente com o aumento da velocidade desde $V=0$, apresentando, portanto, derivada negativa em $V=0$ e, consequentemente, $b<0$. Entretanto, os resultados aqui obtidos com a posterior integração da Eq. (四) independem do sinal de $a$ e $b$.

A força de atrito ao rolamento surge devido ao fato de que nem o corpo nem a superfície são perfeitamente rígidos. Para casos em que o corpo que rola se deforma e a superfície não, $R$ pode ser modelada por [G]

$$
R=\mu_{r}\left(W-F_{S}\right)
$$

em que $\mu_{r}$ é o coeficiente de atrito ao rolamento e $W-F_{S}$ é a força normal, resultante da diferença entre o peso total da aeronave e a força de sustentação durante a decolagem.

As forças de arrasto e sustentação nas Eqs. (『) e (四) tem modelagem clássica [ए]] na forma

$$
\begin{gathered}
F_{A}=\frac{1}{2} \rho\left(V+U_{0}\right)^{2} C_{\text {Atot }} S, \\
F_{S}=\frac{1}{2} \rho\left(V+U_{0}\right)^{2} C_{S} S,
\end{gathered}
$$

em que $\rho$ é a massa específica do ar, $C_{\text {Atot }}$ é o coeficiente de arrasto total, $C_{S}$ é o coeficiente de sustentação e $S$ é a área máxima projetada vertical das duas asas (Apêndice A).

As Eqs. (回) e (四) são oriundas da análise dimensional. Os coeficientes $C_{\text {Atot }}$ e $C_{S}$ em escoamentos subsônicos dependem da geometria e rugosidade do corpo, do ângulo de ataque e do número de Reynolds, Re (Apêndice A). Para uma dada geometria e ângulo de ataque tem-se portanto $C_{\text {Atot }}=C_{\text {Atot }}(\mathrm{Re})$ e $C_{S}=C_{S}(\mathrm{Re})$, o que implica em $C_{A t o t}=C_{A t o t}(V)$ e $C_{S}=C_{S}(V)$. A seguir analisaremos estas dependências.

\footnotetext{
${ }^{4} \mathrm{Na}$ realidade, a hipótese $b=0$ é usada pela Ref. [ [ [] para simplificar a integral resultante da análise.
} 
A dependência $C_{S}$ com Re é geralmente simples, uma vez que a sustentação de aeronave está quase sempre exclusivamente concentrada nas asas. Em aerofólios comerciais a literatura indica que tal efeito é "modesto" [ए]] e que $C_{S}$ pode ser considerado constante com $V$.

Para $C_{\text {Atot }}$ a situação é mais complicada, uma vez que apenas a menor parte do arrasto está localizado nas asas. Definir, portanto, a dependência de $C_{\text {Atot }}$ com Re passa a ser um estudo de caso, com uma solução diferente para cada geometria de aeronave. Esta variação será, contudo, aqui ignorada também, como se faz na maior parte da literatura aeronáutica [田] e $C_{\text {Atot }}$ será considerado constante.

Considerações semelhantes aplicam-se a $\mu_{r}$. A Eq. (四) sugere um valor constante de $\mu_{r}$ para cada par de materiais, mas sabe-se que o atrito depende além disso da velocidade, do torque aplicado às rodas e do seu diâmetro [Q]. A fim de simplificar o problema, apenas a dependência com os materiais será mantida aqui.

Uma explicação final é necessária antes de prosseguirmos com os cálculos. Como a sustentação está praticamente toda concentrada nas asas, a superfície de referência, $S$, aparece naturalmente como a máxima área projetada das asas (Apêndice A). O arrasto, por sua vez, possui vários componentes devidos às diversas partes da aeronave e à interação entre elas, cada um associado, em principio, a uma área de referência diferente. É normal, entretanto, modelar o força de arrasto total através da Eq. (1), referenciando todos os componentes do coeficiente de arrasto à área $S$.

Em geral, divide-se o arrasto como

$$
C_{A t o t}=C_{A \infty}+C_{A i}+C_{A p},
$$

em que o coeficiente $C_{A \infty}$ representa o arrasto da asa infinita, $C_{A i}$ o arrasto induzido e $C_{A p}$ o arrasto parasita (Apêndice A). $\boldsymbol{\Phi}^{\mathbf{T}}$

Asas infinitas são modeladas ignorando as pontas e apresentam um coeficiente arrasto quase tão pequeno quanto placas planas semi-infinitas. Asas reais apresentam uma extremidade em que o ar está livre para passar da zona de alta pressão (sob as asas) para a zona de baixa pressão (sobre as asas) criando vórtices geradores de atrito. Este efeito é modelado pelo coeficiente de arrasto induzido. O modelo matemático clássico [3,, 0$]$ aproveita para incluir neste coeficiente o efeito da interferência entre as asas e a fuselagem e também o efeito solo (Apêndice A). O coeficiente fica

$$
C_{A i}=\frac{\phi C_{S}^{2}}{\pi e_{0} R A}
$$

Aqui $e_{0}$ é o fator de eficiência de Oswald (Apêndice A), que quantifica o efeito da interferência, $\phi$ é o fator que quantifica o efeito solo e $R A$ é a razão de aspecto da asa (Apêndice A). O valor de $e_{0}$ é considerado constante e encontra-se no intervalo $0,6 \leq e_{0} \leq 0,9$ variando conforme o projeto da aeronave. O parâmetro $\phi$ depende do posicionamento das asas ${ }^{\mathbb{0}}$ na aeronave e é modelado [3, 四] por

$$
\phi=\frac{(16 \cdot h / b)^{2}}{1+(16 \cdot h / b)^{2}},
$$

em que $h$ é a distancia vertical média entre as asas e a pista e $b$ é a envergadura das asas (Apêndice A).

O coeficiente de arrasto parasita deve ser calculado em referência à área planificada das asas, $S$, para que

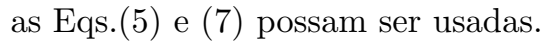

Para finalmente integrar a Eq. (『) falta apenas definir seu limite de integração superior, $V_{d}$. A velocidade mínima em que a aeronave pode se manter no ar, conhecida como velocidade de estol (Apêndice A) pode ser obtida igualando-se seu peso à força de sustentação máxima,

$$
W=\frac{1}{2} \rho\left(V_{\text {estol }}+U_{0}\right)^{2} C_{S \max } S .
$$

Desta equação segue-se que a velocidade de estol relativa ao vento é $V_{\text {estol }}+U_{0}=\left(2 W / \rho C_{S \max } S\right)^{1 / 2}$. A prática aeronáutica, concretizada através das normas de segurança [ $\square]$, estabelece que a aeronave decole a 120\% desta velocidade mínima, portanto,

$$
V_{d}=1,2 \sqrt{\frac{2 W}{\rho C_{S \max } S}}-U_{o} .
$$

Substituindo as Eqs. (四)-(四) na Eq. (『) segue-se

$$
S_{d}=\frac{W}{g} \int_{0}^{V_{d}} \frac{V d V}{A V^{2}+B V+C},
$$

em que

$$
\begin{gathered}
A=a-0,5 \rho\left(C_{\text {Atot }}-\mu_{r} C_{S}\right) S, \\
B=b+2 a U_{0}-\rho U_{0}\left(C_{\text {Atot }}-\mu_{r} C_{S}\right) S, \\
C=T_{0}-\mu_{r} W+a U_{0}^{2}+b U_{0}- \\
0,5 \rho U_{0}^{2}\left(C_{\text {Atot }}-\mu_{r} C_{S}\right) S,
\end{gathered}
$$

em que $A, B$ e $C$ são independentes de $V$, devido às considerações feitas anteriormente e $V_{d}$ é dada pela Eq. (एШ) .

O resultado da integral na Eq. (एव) encontra-se tabelado em textos clássicos como [एँ]

$$
\begin{aligned}
S_{d} & =\frac{W}{2 g A}\left[\ln \left|\frac{A V_{d}^{2}+B V_{d}+C}{C}\right|\right. \\
& \left.-\frac{2 B}{\sqrt{-\Delta}}\left(\arctan \frac{2 A V_{d}+B}{\sqrt{-\Delta}}-\arctan \frac{B}{\sqrt{-\Delta}}\right)\right],
\end{aligned}
$$

\footnotetext{
${ }^{5}$ Alguns textos [ [3] agrupam o arrasto da asa infinita e o parasita em apenas um termo chamado arrasto parasita. Em nossa opinião isso obscurece o assunto, pois o termo "parasita" sugere um arrasto não essencial ao vôo e isto as asas certamente não são. Utilizamos, portanto, a notação da Ref. [0]

${ }^{6}$ Tradicionalmente classificado como asas baixas, médias ou altas (Apêndice A).
} 
para $-\Delta=4 A C-B^{2}>0 \mathrm{e}$

$$
\begin{aligned}
S_{d} & =\frac{W}{2 g A}\left[\ln \left|\frac{A V_{d}^{2}+B V_{d}+C}{C}\right|\right. \\
& \left.-\frac{B}{\sqrt{\Delta}}\left(\ln \frac{2 A V_{d}+B-\sqrt{\Delta}}{2 A V_{d}+B+\sqrt{\Delta}}-\ln \frac{B-\sqrt{\Delta}}{B+\sqrt{\Delta}}\right)\right],
\end{aligned}
$$

para $\Delta=B^{2}-4 A C>0$.

A integral anterior seria simplificada caso o vento frontal fosse desconsiderado e a tração (Eq. (国) ) descrita por $T=a V^{2}+c$. Neste caso teríamos $B=0 \mathrm{e}$

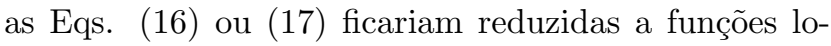
garítmicas como na Ref. [ర]].

Um texto bastante tradicional na área de tabelas de integrais, [․:3], apresenta apenas a Eq. (마) como solução da integral da Eq. (एా), sem mencionar para quais valores de $\Delta$ ela se aplica. Intrigados com a diferença entre os textos e com a possibilidade de que uma equação com argumentos complexos, no caso $\sqrt{-\Delta}$ com $-\Delta<0$, pudesse fornecer valores reais para $S_{d}$, fomos investigar a questão.

Para nossa surpresa, implementando a Eq. ([ய]) em Matlab ${ }^{\circledR}$ obteve-se um resultado real. Depois de alguma álgebra e utilizando identidades trigonométricas para o arctans de números complexos e para a soma ou diferença do arctans de números complexos chegamos exatamente à Eq. (마). Por simplicidade, contudo, optamos por apresentar o resultado final de nossa analise na forma das Eqs. (미) e (ㅁ), como na Ref. [ए]] .

Chamamos a atenção, porém, para o fato que, desde que se disponha de um programa ou calculadora capaz de lidar com números complexos, a Eq. (미) descreve a solução do problema independente do valor de $\Delta$.

Resta, então, analisar o caso $\Delta=0$. Pode-se entender o significado físico deste caso pensando da seguinte forma. A Eq. (四) exige que $A V^{2}+B V+C \neq 0$. Isso apenas diz que a decolagem não ocorre se a soma das forças horizontais for nula, pois $A V^{2}+B V+C=$ $\left(T-F_{A}-R\right)$.

Entendendo então $F_{x}=A V^{2}+B V+C$ como o somatório de forças horizontais no sentido da decolagem, fica claro que a parábola não pode ter raízes no domínio do problema (nem assumir valores negativos, aliás). Neste caso, $\Delta \neq 0$ apenas afirma que $F_{x}(V)$ não possui raiz única no domínio do problema, o que é redundante em relação a ela não possuir raízes. A existência de uma ou mais velocidades em que $F_{x}=0$ no intervalo $\left[0, V_{d}\right]$ implicaria na aeronave parar de acelerar, não atingindo, portanto a velocidade de decolagem.

\footnotetext{
${ }^{7}$ Nem todo pouso é realizado desta maneira. Os pousos em porta-aviões, por exemplo, são feitos com "reserva" de motor caso a aeronave precise arremeter (Apêndice A) por motivos de segurança.
}

\section{Análise do pouso}

\subsection{O processo de pouso}

As forças atuantes durante o pouso são as mesmas da decolagem (Fig. 四) exceto pela tração, que é considerada nula, uma vez que o piloto em geral reduz o motor para a condição de marcha lenta (Apêndice A) ao tocar a pista. $\mathbf{\square}$

Pousar uma aeronave é essencialmente desacelerála da velocidade de toque na pista, $V_{p}$, até o repouso. $\mathrm{Na}$ prática, novamente, existem alguns detalhes e aeronaves de diferentes categorias pousam de maneiras diferentes.

Em aeronaves de grande porte, a aproximação da pista é feita a uma razão de descida (Apêndice A) constante, com o GMP em regime de baixa tração e mantendo a velocidade acima da de estol. Ao cruzar a cabeceira da pista (Apêndice A), o piloto reduz ainda mais a potência do motor (em geral colocando-o em marcha lenta) e levanta o nariz da aeronave para aumentar o arrasto e a sustentação, simultaneamente reduzindo a velocidade e a razão de descida. A aeronave é mantida nesta situação por uns instantes, "planando" a alguns centímetros da pista, perdendo velocidade e sustentação até o momento do toque. Neste ponto, a norma [प]] recomenda $V_{p}=1,3 V_{\text {estol }}$, por razões de segurança.

Logo após o toque, o piloto deve manter a aeronave em atitude de nariz elevado por mais alguns instantes, para evitar choques e, portanto, esforços excessivos sobre o trem de pouso dianteiro. Com a posterior redução da velocidade, o nariz se abaixa, reduzindo o ângulo de ataque e a sustentação. Inicia-se a desaceleração com todas as rodas em contato com a pista, que prossegue até a imobilidade. Nesta fase, geralmente se utiliza algum procedimento de frenagem para reduzir a distância até a imobilidade.

Aeronaves pequenas realizam procedimento muito semelhante, mas podem tocar a pista a velocidades mais próximas de $V_{\text {estol }}$ e não possuem dispositivos de frenagem sofisticados.

O pouso, assim como a decolagem, é realizado contra o vento, para que a sustentação necessária seja obtida em menores velocidades relativas aeronave-pista. Isso garante maior controle ao piloto e também aumenta a força de arrasto, reduzindo a distância de pouso.

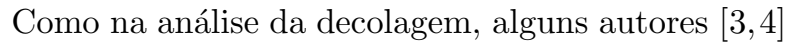
aproximam a integral utilizando valores médios para as forças, calculados a $70 \%$ da velocidade de pouso, enquanto outros [四, [6] resolvem a integral numericamente.

\subsection{A Equação do movimento}

Para a análise matemática do pouso, retoma-se a análise da seção anterior com $T=0$ e inclui-se uma 
força de frenagem, $F_{F}$. A integral da Eq. (『) torna-se

$$
\begin{aligned}
S_{p} & =\frac{W}{g} \int_{V_{p}}^{0} \frac{V d V}{-F_{A}-R-F_{F}} \\
& =\frac{W}{g} \int_{0}^{V_{p}} \frac{V d V}{F_{A}+R+F_{F}}
\end{aligned}
$$

em que $S_{p}$ é a distância percorrida desde o toque na pista até a parada e $V_{p}$ é a velocidade de toque.

Modelar matematicamente a força de frenagem não é possível, uma vez que o piloto pode acionar o freio da maneira que lhe for conveniente. Pode-se, contudo, estabelecer um limite máximo para a frenagem:

$$
F_{F}=\mu_{e}\left(W-F_{S}\right)
$$

em que $\mu_{e}$ é o coeficiente de atrito estático entre os pneus e a pista e $W-F_{S}$ é a força normal. Qualquer valor de $F_{F}$ maior do que este faz as rodas travarem, reduzindo a eficiência da frenagem, uma vez que o coeficiente de atrito dinâmico é menor que o estático. Isso é evitado pelo sistema ABS (Apêndice A) ou pela experiência do piloto.

A semelhança formal entre as Eqs. (田) e (एप) mostra que o uso dos freios no limite máximo atua como um incremento ao atrito de rolamento. Porém, enquanto valores típicos de $\mu_{r}$ para pneus de carro sobre asfalto situam-se em torno de 0,01 , os de $\mu_{e}$ ficam em torno de $0,9[\mathrm{G}]$. Isso mostra porque o uso de freios é tão eficiente no procedimento de pouso. ${ }^{\nabla}$

Substituindo a Eq. (ㅁ) na Eq. (ष) e usando para as outras forças a mesma modelagem anterior tem-se

$$
S_{p}=\frac{W}{g} \int_{0}^{V_{p}} \frac{V d V}{A_{p} V^{2}+B_{p} V+C_{p}},
$$

em que

$$
\begin{gathered}
A_{p}=0,5 \rho\left(C_{\text {Atot }}-\left(\mu_{r}+\mu_{e}\right) C_{S}\right) S, \\
B_{p}=\rho U_{0}\left(C_{\text {Atot }}-\left(\mu_{r}+\mu_{e}\right) C_{S}\right) S, \\
C_{p}=\left(\mu_{r}+\mu_{e}\right) W \\
+0,5 \rho U_{0}^{2}\left(C_{\text {Atot }}-\left(\mu_{r}+\mu_{e}\right) C_{S}\right) S, \\
V_{p}=1,3 \sqrt{\frac{2 W}{\rho C_{S \max } S}}-U_{o} .
\end{gathered}
$$

A integral da Eq. (四) é formalmente idêntica à da
Eq. (ए2) trocando $V_{d}$ por $V_{p}$. Logo,

$$
\begin{aligned}
S_{d}=\frac{W}{2 g A_{p}}\left[\ln \left|\frac{A_{p} V_{p}^{2}+B_{p} V_{p}+C_{p}}{C}\right|\right. \\
-\frac{2 B}{\sqrt{-\Delta_{p}}}\left(\arctan \frac{2 A_{p} V_{p}+B_{p}}{\sqrt{-\Delta_{p}}}-\right. \\
\left.\left.\arctan \frac{B_{p}}{\sqrt{-\Delta_{p}}}\right)\right],
\end{aligned}
$$

$$
\begin{aligned}
& \operatorname{para}-\Delta_{p}=4 A_{p} C_{p}-B_{p}^{2}>0 \mathrm{e} \\
& S_{d}=\frac{W}{2 g A}\left[\ln \left|\frac{A_{p} V_{p}^{2}+B_{p} V_{p}+C_{p}}{C_{p}}\right|\right. \\
& -\frac{B_{p}}{\sqrt{\Delta_{p}}}\left(\ln \frac{2 A_{p} V_{p}+B_{p}-\sqrt{\Delta_{p}}}{2 A_{p} V_{p}+B_{p}+\sqrt{\Delta_{p}}}\right. \\
& \left.\left.-\ln \frac{B_{p}-\sqrt{\Delta_{p}}}{B_{p}+\sqrt{\Delta}}\right)\right],
\end{aligned}
$$

para $\Delta_{p}=B_{p}^{2}-4 A_{p} C_{p}>0$, com as constantes $A_{p}, B_{p}$ e $C_{p}$ dadas pela Eqs. (四)-(国) e $\mu_{e}=0$ caso não haja aplicação de freios.

A interpretação física do caso em que $4 A_{p} C_{p}-B_{p}^{2}=$ 0 é semelhante ao caso da decolagem. A parábola aqui representa a força resistiva total durante o pouso, estritamente negativa. A existência de uma ou mais velocidades em que $F_{x}=0$ no intervalo $\left[V_{p}, 0\right]$ implicaria na aeronave parar de desacelerar, não atingindo, portanto o repouso.

\section{Medidas experimentais da tração}

Para obter resultados numéricos para as distâncias de

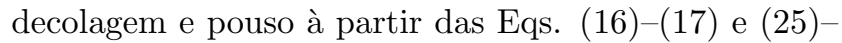
(며), valores de $a, b$ e $T_{0}$ na Eq. ([) precisam ser conhecidos para o GMP da aeronave avaliada. No caso da aeronave comercial, tais dados foram obtidos da literatura [ []. Para o VANT, foi construída uma balança de tração (Apêndice A) de baixo custo, como detalhado a seguir, e testado o GPM usado pela equipe.

A uma base de madeira (Fig. [1) foram fixados dois suportes verticais: um fixo, com dois apoios, para suportar uma célula de carga de ponto único, HBM modelo PW6DC3, e outro, pivotado em um apoio, para suportar o GMP. O GMP foi ligado à celula de carga através de um cabo de aço de comprimento apenas suficiente para manter o suporte pivotado na vertical durante as medições. Foi instalado um tubo de pitot da Eagle Tree Systems, conectado a um sensor de pressão diferencial da Freescale Semiconductor modelo MPXV5004DP em um suporte lateral do conjunto para

\footnotetext{
${ }^{8}$ Claro que, neste sentido, também é útil aumentar-se o valor da força normal. Isso é feito pelos spoilers (Apêndice A). Matematicamente, eles agem na Eq. (ष) aumentando todos os termos no denominador da integral.

${ }^{9} \mathrm{Na}$ verdade a balança foi usada para definir o melhor GMP para a competição. Os resultados aqui apresentados referem-se ao conjunto escolhido.
} 
realizar medidas da velocidade relativa do ar. Os sinais de saída da celula de carga e do sensor de pressão foram adquiridos por um microcontrolador de 8 bits da marca Atmel modelo ATmega328 e transmitidos a um microcomputador onde os mesmos foram convertidos para velocidade e tração e salvos através do software Labview ${ }^{\circledR}$.

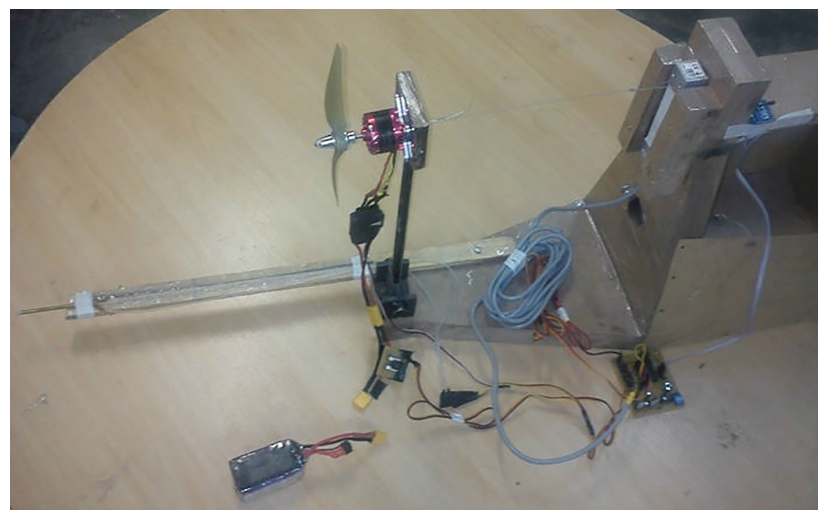

Figura 3 - Balança de tração.

O GMP típico do VANT usado na competição Aerodesign é constituído por motor elétrico, hélice, Electronic Speed Control (ESC) e bateria. No experimento foi utilizado o motor elétrico marca Turnigy, modelo 3632 , de $1500 \mathrm{Kv}$, a bateria tipo de $1.300 \mathrm{mAh}$, tipo LiFePO4 3S e um ESC marca HobbyKing de 50 A, com uma hélice bi-pá marca APC, medida 12x6.

O conjunto descrito foi fixado a uma base construída em perfis metálicos e estes foram presos à carroceria de uma caminhonete, posta em movimento para simular o

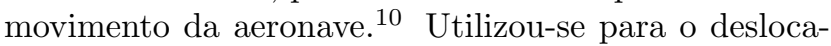
mento da caminhonete uma pista de pouso, a mesma em que os testes de voo eram realizados. O experimento foi realizado em um dia sem vento, variando a velocidade da caminhonete de 0 a $80 \mathrm{~km} / \mathrm{h}$ aproximadamente. Foram tomados os cuidados possíveis para só registrar medidas em trechos não acelerados do movimento.

Os dados de tração brutos do teste, $T_{h}$, foram padronizados para o nível do mar, $T_{0}$, como recomenda a literatura [3]. Uma vez que a tração é diretamente proporcional à massa específica, foi utilizada a relação $T_{0}=T_{h}\left(\rho_{0} / \rho_{h}\right)$, em que $\rho_{0}=1,01325.10^{5}$ Pa e $\rho_{h}$ foi medido a $2 \mathrm{~m}$ de altura no local com uma estação meteorológica portátil marca Kestrel, modelo 4000. Os resultados foram recorrigidos para a altitude aproximada da competição $(1.200 \mathrm{~m})$ utilizando-se novamente a relação anterior e são mostrados na Fig. 由.

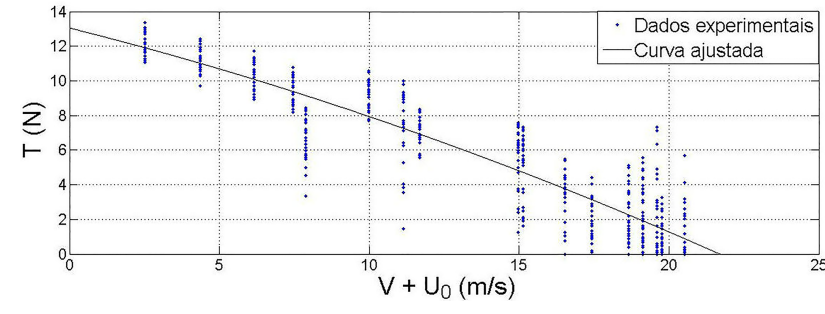

Figura 4 - Variação da tração do conjunto motopropulsor do VANT em função da velocidade relativa do vento.

Percebe-se que, como previsto, os pontos experimentais são bem descritos por uma parábola com $a<0$ e $b<0$ (Eq. (国)). Os coeficientes da parábola ajustada pelo método dos mínimos quadrados são

$$
\begin{gathered}
a=-0,00572 \mathrm{~N} /(\mathrm{m} / \mathrm{s})^{2}, \\
b=-0,355 \mathrm{~N} /(\mathrm{m} / \mathrm{s}), \\
T_{0}=14,387 \mathrm{~N} .
\end{gathered}
$$

O espalhamento relativamente grande dos resultados experimentais se deve provavelmente à baixa resolução do sensor acoplado ao tubo de pitot. Outro fator a se considerar são possíveis períodos de aceleração (ou desaceleração) da caminhonete, que acrescenta (ou subtrai) uma força associada ao sistema não-inercial de referência. Para as finalidades didáticas do presente estudo, no entanto, os resultados da Fig. 四 serão considerados suficientemente precisos e esforços serão realizados para melhorar a qualidade das medidas no futuro.

Para os dados do Cessna obteve-se

$$
\begin{gathered}
a=0,0353 \mathrm{~N} /(\mathrm{m} / \mathrm{s})^{2}, \\
b=-18,602 \mathrm{~N} /(\mathrm{m} / \mathrm{s}), \\
T_{0}=2.436,972 \mathrm{~N},
\end{gathered}
$$

com $a>0$ e $b<0$, conforme previsto.

\section{Resultados - decolagem}

Para testar os resultados das Eqs. (ㅁ]) e (एच) são necessários valores de $S_{d}, W, C_{A t o t}, C_{S}, C_{S \max }, \mu_{r}$ e $S$ além das constantes $a, b$ e $T_{0}$. Para testar as Eqs. (2.5) e (区ש), ainda é preciso conhecer $\mu_{e}$. Para demonstrar a ampla faixa de aeronaves à qual a metodologia se aplica, $S_{d}$ foi calculado para dois modelos de uma aeronave comercial, o Cessna 172 Skyhawk modelos S e N, e para um dos VANTs usado na competição Aerodesign 2014. A tabela 1 resume os valores utilizados. No caso do Cessna eles foram obtidos da literatura [0, ㅁ,, ㅁ. ] e no caso do VANT vieram dos cálculos da própria equipe. Infelizmente não foi possível obter resultados para $S_{p}$.

Na Tabela $1, S_{d}$ calc. representa o resultado da Eq. (ㅁ) e $S_{d}$ calc. [3] representa o valor calculado pelo método tradicional [3]. Os valores de $\rho$ utilizados são para o nível do mar no caso do Cessna e para a altitude da competição, via atmosfera padrão, para o protótipo.

\footnotetext{
${ }^{10}$ Claro que um túnel de vento seria o equipamento adequado para este fim mas, como mencionado, tratava-se de um projeto de baixa disponibilidade financeira.
} 
Tabela 1 - Distância de decolagem.

\begin{tabular}{lccc}
\hline & Cessna N & Cessna S & VANT \\
\hline$W / g$ & 1043 & 1156,6 & 3,13 \\
$C_{A T o t}$ & 0,0320 & 0,0320 & 0,0646 \\
$C_{S}$ & 0,41 & 0,41 & 0,44 \\
$C_{S \max }$ & 2,1 & 2,1 & 1,418 \\
$S$ & 16,07 & 16,07 & 0,340 \\
$\mu_{r}$ & 0,03 & 0,03 & 0,11 \\
$S_{d}$ medido & 219 & 293 & 45 \\
$S_{d}$ calc. & 215,92 & 275,40 & 36,31 \\
$S_{d}$ calc. [] & 215,87 & 275,26 & 37,18 \\
\hline
\end{tabular}

Verfica-se que os valores calculados pela Eq. (미) subestimam as medidas do Cessna em apenas 1,4\% (modelo $\mathrm{N}$ ) e $6,0 \%$ (modelo $\mathrm{S}$ ), praticamente o mesmo resultado obtido calculado pela Ref. [3]. Para o VANT, os resultado da Eq. (ㅁ) e da Ref. [3] apresentam-se também muito próximos, ambos subestimando $S_{d}$ medido em $19,3 \%$. Contudo, $S_{d}$ medido teve que ser estimado neste caso. $\mathrm{Na}$ ausência de um sistema adequado de telemetria não se pode garantir a arfagem a $V=1,2 V_{\text {estol }}$ (ver Eq. (Ш⿴囗⿰丿㇄口)), sendo a tendência do piloto nesse caso alongar a corrida de decolagem por razões de segurança.

A título de ilustração alguns parâmetros foram variados para os dados do VANT na Eq. (미) e sua influencia foi representada nas figuras a seguir.

A Fig. 1 mostra a curva velocidade versus deslocamento obtida da Eq. (ㅍ) usando diferentes valores de $V_{d}$. Pelo método tradicional [3] não é teoricamente aconselhável traçar a curva anterior, uma vez que a média utilizada na integral é proposta apenas para o intervalo $\left[0, S_{d}\right]$.

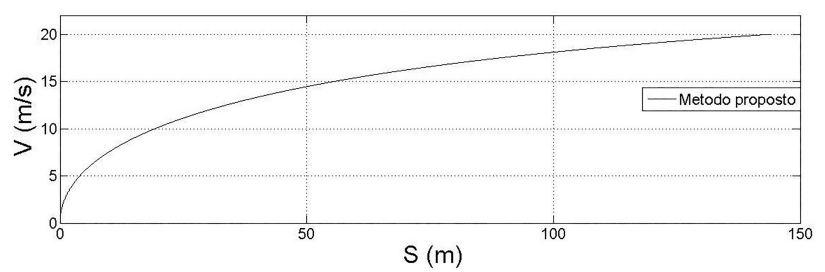

Figura 5 - Velocidade vs. deslocamento durante a corrida de decolagem.

A Fig. [ 6 mostra a dependência da distancia de decolagem com a velocidade do vento frontal. Além da esperada redução de $S_{d}$ com $U_{0}$, ela mostra que a aeronave "decola" sozinha uma vez atingida certo valor de $U_{0}$. Para que tal decolagem ocorra na prática, a aeronave precisa estar com o nariz levantado, imitando o efeito de arfagem ao final da corrida. Nesse caso, porém, ela começaria a flutuar antes do previsto na Fig. [6, devido ao fator de segurança de 1,2 na Eq. (ШD).

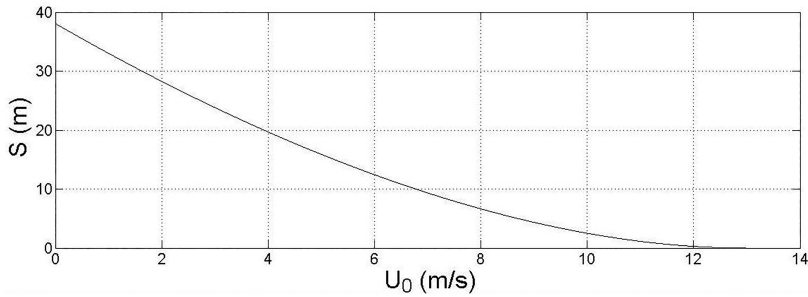

Figura 6 - Distância de decolagem. Variação com a velocidade do vento frontal.

Por fim, a Fig. $\square$ ilustra a dependência da distancia de decolagem com o peso total da aeronave. Como na prática o comprimento de pista é limitado, este é o resultado usado para escolher a carga máxima a ser transportada.

A figura mostra a esperada tendência de que $S_{d} \rightarrow$ $\infty$ para um determinado peso, isto é, mostra o máximo de carga com a qual a aeronave consegue decolar. A condição para que isso ocorra é que o somatório de forças horizontais, Eq. (四), iguale-se a zero antes que seja atingida a velocidade em que o peso é equilibrado pela sustentação, ou seja,

$$
T-F_{A}-R=0,
$$

para

$$
W_{\max }=\frac{1}{2} \rho\left(V+U_{0}\right)_{\max }^{2} \frac{C_{S \max }}{1,2^{2}} S
$$

em que o fator $1,2^{2}$ foi deixado explícito propositadamente para mostrar que se trata do coeficiente de segurança mencionado na Eq. (प]).

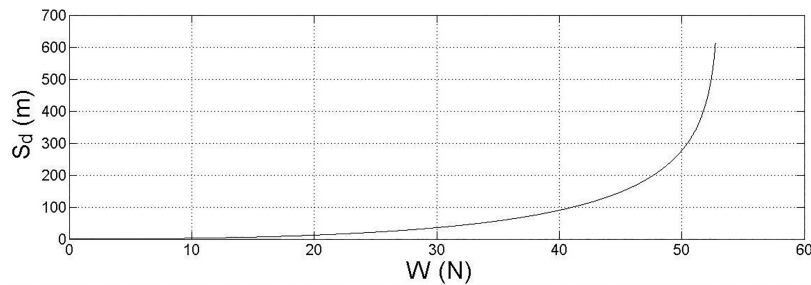

Figura 7 - Distância de decolagem. Variação com o peso total da aeronave.

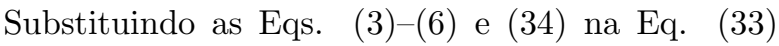
resulta uma equação do segundo grau cuja menor raiz fornece o peso limite de decolagem

$$
W_{\max }=\frac{1}{2} \rho \frac{C_{\text {Smax }}}{1,2^{2}} S\left[\frac{-b-\sqrt{b^{2}-4 A^{*} T_{0}}}{2 A^{*}}\right]^{2},
$$

em que

$$
A^{*}=a-\frac{\rho}{2}\left(C_{A t o t}-\mu_{r} C_{S}+\mu_{r} \frac{C_{S \max }}{1,2^{2}}\right) S .
$$

Evidentemente a equação anterior é válida tanto para o VANT quanto para as aeronaves comerciais. Para o VANT ela fornece $W_{\max }=52,78 \mathrm{~N}$. 


\section{Conclusões}

O presente trabalho apresenta uma análise das corridas de decolagem e pouso em uma aeronave com forças dependentes da velocidade. Foram consideradas as forças aerodinâmicas de arrasto e sustentação, o peso total da aeronave, bem como o atrito de rolamento e a força de frenagem máxima (no pouso). A análise assumiu:

- Dependência quadrática da tração com a velocidade, mas com $b \neq 0$;

- Vento de nariz ou de cauda;

- Arrasto, sustentação e atrito de rolamento modelados da forma tradicional, com coeficientes constantes;

- Efeitos solo, de interferência entre asa e fuselagem e de ponta de asa;

- Frenagem máxima, porém sem travamento das rodas.

Menos restrições foram impostas durante a análise do que na literatura tradicional, em particular em relação ao comportamento da tração.

O resultado da análise mostrou-se bastante robusto, sendo aplicado com êxito uma aeronave comercial e a um VANT em escala reduzida. Para comparar os resultados da análise com dados do VANT, foi projetada e construída uma balança de tração de baixo custo, onde foi levantada a curva de tração do GPM utilizado. Estudos estão sendo realizados no sentido de melhorar a construção da balança de tração e de construir um túnel de vento onde ela possa ser testada.

\section{Agradecimentos}

Os autores agradecem aos acadêmicos Alexandre Condé Leite, Vinícius Luiz de Souza e Edgar Luiz Vanier pelo auxílio na construção e instalação do sistema de aquisição de dados da balança tração.

\section{Apêndice A - Glossário de termos ae- ronáuticos}

Ângulo de ataque: ângulo entre a corda do aerofólio e o escoamento não perturbado

Área alar: o mesmo que área máxima projetada vertical das asas

Área máxima projetada vertical das asas: área das duas asas da aeronave projetada em um plano definido por seus eixos longitudinal e lateral

Arfagem: ato de levantar o nariz da aeronave. Movimento de rotação em torno do eixo lateral (passando pelas asas)
Arrasto da asa infinita: força de arrasto sobre uma hipotética asa semi-infinita, i.e., sem pontas. Nos aerofólios modernos é quase tão pequeno quanto o arrasto sobre uma placa plana semi-infinita.

Arrasto induzido: asas reais (com pontas) apresentam uma extremidade em que o ar está livre para passar da zona de alta pressão (sob as asas) para a zona de baixa pressão (sobre as asas) criando vórtices geradores de atrito. Este efeito é modelado acrescentando-se a força de arrasto induzido à força de arrasto da asa infinita.

Arrasto parasita: força de arrasto devido a todos os demais componentes da aeronave, exceto as asas

Arremeter: ato de abortar o pouso depois de aproximar-se ou de tocar a pista. É feito elevando-se a potência do motor ao máximo e voltando a ganhar altura sem ter chegado ao repouso. Realizado em situações de emergência para evitar pousos possivelmente catastróficos.

Asas baixas, médias ou altas: indica a forma como as asas são fixadas à fuselagem, respectivamente em sua parte inferior, mediana ou superior. Cada tipo possui características específicas que as tornam mais adequadas a uma determinada função. Por exemplo, asas altas facilitam a retirada de carga e ampliam a visão do piloto durante o pouso

Balança de tração: no presente contexto, é uma bancada experimental utilizada em ensaios de grupo moto-propulsores para medir a força exercida pela hélice sobre a aeronave em movimento

Bordo de ataque: ponto do aerofólio mais a montante do escoamento. Formato arredondado

Bordo de fuga: ponto do aerofólio mais a jusante do escoamento. Formato afilado

Cabeceira da pista: início físico da pista de pouso e decolagem. Eventualmente muda de lado para garantir que as aeronaves pousem sempre contra o vento

Coeficiente de arrasto: força de arrasto adimensional (ver Eq. (回)). Pode ser obtido, entre outros métodos, pelo Teorema de Vaschy-Buckingham da análise dimensional. A força é adimensionalizada pelo produto da pressão dinâmica pela área alar

Coeficiente de sustentação: força de sustentação adimensional (ver Eq. (因)). Idem coeficiente de arrasto

Corda: linha reta que une os bordos de ataque e de fuga em um aerofólio. Pode ser constante, em asas retangulares, ou variável, em asas de outros tipos (trapezoidal, reto-trapezoidal, elíptica, etc.) 
Corda média: valor médio da corda em asas com geometria não retangular. É calculado pela relação entre a área de uma asa e seu comprimento

Efeito solo: a proximidade com o solo, durante pousos e decolagens, limita fisicamente o tamanho dos vórtices de ponta de asa, reduzindo o arrasto induzido

Envergadura das asas: comprimento total do par de asas, de ponta a ponta

Fator de eficiência de Oswald: valor que quantifica matematicamente o efeito da interferência aerodinâmica entre as asas e a fuselagem

Força de arrasto: no escoamento de um fluido ao redor de um corpo qualquer, força de arrasto é a componente da força total originada pelo fluido sobre o corpo na direção do escoamento não perturbado pela presença do corpo

Força de sustentação: idem força de arrasto, porém na direção perpendicular ao escoamento não perturbado. Em muitos textos clássico o aparecimento da força de sustentação é explicado via Eq. de Bernoulli mas, como observa [ए6], este raciocínio é incompleto e a explicação completa repousa na curvatura das linhas de corrente do escoamento ao circular o aerofólio. Outra interessante interpretação para a sustentação aparece na frase "A sustentação de uma asa equivale à variação do fluxo do impulso vertical causada pela asa", do mesmo autor

Grupo moto-propulsor (GMP): como o nome indica, é o conjunto formado pelo motor e pelo propulsor da aeronave. Chama-se de GPM o conjunto motor-hélice em aviões convencionais, turbina-hélice em turboélices e apenas turbina em turbojatos. O mesmo motor usando hélices diferentes pode gerar uma tração diferente

Marcha lenta: condição em que o motor gera potência apenas para continuar seu próprio funcionamento e manter operacionais os sistemas vitais das aeronave como fornecimento de energia elétrica, pressão nos aturadores hidráulicos, pressurização da cabine, etc. Não há sobra de potência útil para o vôo

Número de Reynolds: número adimensional que expressa a razão entre as forças de inércia e de atrito viscoso em um escoamento: $R e=\rho V c / \mu$, em que $c$ é a corda média e $\mu$ a viscosidade do fluido. Pode ser obtido, entre outros métodos, pelo Teorema de Vaschy-Buckingham da análise dimensional.

Ponto de estol: a função $C_{S}=C_{S}(\alpha)$ para aerofólios típicos aumenta até um valor máximo e depois cai bruscamente. O ponto de máximo é conhecido como ponto de estol (ver Fig. 『) e sinaliza a rápida perda de sustentação. Exceto em condições extraordinárias (como perseguições aéreas) não se voa além do ponto de estol

Razão de aspecto da asa: relação entre o comprimento e a corda média de uma asa

Razão de descida: taxa de variação da altitude da aeronave em relação ao tempo durante a aproximação da pista

Sistema ABS: sistema de frenagem que evita o bloqueio das rodas, impedindo que o rolamento dos pneus se transforme em escorregamento

Spoiler: ou destruidor de sustentação, é um aparato mecânico que reduz bruscamente a força de sustentação sobre um aerofólio sem variar seu ângulo de ataque. Tipicamente é uma superfície móvel que se eleva na parte superior das asas. Utilizado para melhorar a frenagem durante o pouso e, eventualmente, para aumentar a razão de descida e reduzir a velocidade em vôo. Também aumenta o arrasto

VANT: veículo aéreo não tripulado

Velocidade de estol: velocidade mínima em que a aeronave pode se manter no ar, obtida com o $C_{S}$ do ponto de estol

\section{Referências}

[1] SAE Brasil, disponível em http://www.saebrasil. org.br/eventos/programasestudantis/aero20147 aero.aspy, acesso em 4/10/2014.

[2] P.K. Kundu, I.M. Cohen and D.R. Dowling, Fluid Mechanics (Academic Press, Boston, 2011).

[3] L.E.M.J. Rodrigues, Fundamentos da Engenharia Aeronáutica (Cengage Learning, São Paulo, 2013).

[4] J.D. Anderson, Aircraft Performance and Design (WCB/McGraw Hill, Boston, 1998).

[5] C.D. Perkings and R.E. Hage, Airplane Performance Stability and Control (Wiley, Michigan, 1949 ).

[6] J. Roskam and C.E. Lan, Airplane Aerodynamics and Perfomance (DAR Corporation, Lawrence, 2000).

[7] J.B. Russell, Performance and Stability of Aircraft (Butterworth-Heinemann, Burlington, 1996).

[8] John McIver, Cessna Skyhawk II/100 Performance Assessment (Temporal Images, Melbourne, 2003)

[9] A.V. Andrade-Neto, J.A. Cruz, M.S.R. Miltão e E.S. Ferreira, Revista Brasileira de Ensino de Física 35, 3704 (2013).

[10] F.M. White, Mecânica Dos Fluidos (McGraw Hill, Rio de Janeiro, 2002).

[11] Federal Aviation Regulations, Disponível em "http:// www.flightsimaviation.com/data/FARS/, acesso em $4 / 10 / 2014$ 
[12] M.R. Spiegel, Manual de Fórmulas, Métodos e Tabelas Matemáticas (McGraw-Hill, São Paulo, 1992).

[13] M. Abramowitz and I.A. Stegun, Handbook of Mathematical Functions: With Formulas, Graphs, and Mathematical Tables (Dover Publications, Washington, D.C, 1972).

[14] Cessna Aircraft Company, Cessna Skyhawk 172S, Spe- cification and Description (Cessna, Wichita, 2012).

[15] Cessna Aircraft Company, Pilot's Operational Handbook: Skyhawk Cessna Model 172N (Cessna, Wichita, 1978).

[16] K. Weltner, M. Ingelman-Sundberg, A.S. Esperidiao e P. Miranda, Revista Brasileira de Ensino de Física 23, 429 (2001). 